\title{
Research on the Temporal and Spatial Distribution Characteristics of Thermal Anomalies in Henan Province from 2008 to 2019
}

\author{
Qiang $\mathrm{Ge}^{1}$, Wenju Shen ${ }^{1 *}$, Shenshen $\mathrm{Li}^{2}$ and Kun Cai ${ }^{1}$ \\ ${ }^{1}$ Computer and Information Engineering College, Henan University, Kaifeng, HeNan Province, 475004, China \\ ${ }^{2}$ State Key Laboratory of Remote Sensing Science, Institute of Remote Sensing and Digital Earth, Chinese Academy of Sciences, Beijing, \\ 100020, China
}

\begin{abstract}
Using MODIS standard products, the temporal and spatial distribution characteristics of thermal anomalies in Henan Province in the past 12 years (2008 2019) were studied. The results found that in terms of spatial distribution, thermal anomalies were mostly concentrated in Luohe, Zhumadian, Pingdingshan, Puyang, and Shangqiu. The number of areas under the jurisdiction of Anyang, Hebi, Nanyang and Xinyang is relatively high. On the inter-annual trend, the number of thermal anomalies continued to increase from 2009 to 2013 , with an average annual growth rate of $28.3 \%$, and a continuous decline from 2013 to 2018 . The decline rate was $18.4 \%$, during which the number reached a peak of 5,843 in 2013 . In terms of seasonal changes, the number of summer thermal anomalies is the largest, at 25,361, and thermal anomalies of summer are mostly concentrated in most areas of Zhumadian, Pingdingshan, Puyang and Shangqiu; the number of thermal anomalies in winter is the least, 3974, which are relatively mostly distributed in the mountainous areas of Nanyang and Xinyang. This study helps to understand the forest fires in typical areas in Henan Province, as well as heat caused by straw burning, industrial emissions, etc. Thermal anomalies changes provide technical support for regional disaster prevention and environmental monitoring.
\end{abstract}

\section{Introduction}

The use of thermal infrared remote sensing technology can generate thermal anomaly remote sensing information products that reflect the properties and spatial information of surface objects. Nowadays, these thermal anomaly remote sensing information products have been used in many industries. For example, Sun Shuang et al.1 used thermal anomaly remote sensing products to explore the changing law of the radiation intensity of thermal anomalies and its relationship with industrial energy consumption and pollutant emissions. Jin Quanfeng et al.2 and Yang Xiajie et al.3 Use MODIS thermal anomaly remote sensing products to analyze the total discharge of forest fire pollutants in forest areas and their temporal and spatial distribution; In addition, the use of thermal anomaly remote sensing monitoring technology can also monitor and identify biomass burning. Straw burning is a kind of biomass burning, and it is a common practice in biomass management used in many parts of the world to prepare land for the next round of crop planting4. However, a large amount of straw burning can cause serious harm to the environment. For example, It will cause a waste of resources; it may cause a large area of fire accidents, which poses a great threat to the lives and properties of ordinary people; reduce transportation efficiency and may cause traffic accidents, etc5.

Satellite remote sensing has the advantages of large area coverage, high time frequency, and less restricted conditions. It can replace the traditional time-consuming and inaccurate method of monitoring the open-air burning of straw on artificial ground6. Because MODIS is suitable for responding to the characteristics of rapid and sudden changes in straw burning, it has always been an ideal data source in the monitoring of large-scale straw burning. The research idea of this paper is to select Henan Province as the research area of thermal anomaly points, and use the MOD14/MYD14 secondary standard data products of Henan Province for a total of 12 years from 2008 to 2019 as the data source. The research idea of this paper is to select Henan Province as the research area of thermal anomalies, and the data are derived from the MOD14/MYD14 second-level standard data products of Henan Province from 2008 to 2019, The research idea of this paper is to select Henan Province as the research area of thermal anomalies, and the data source is the MOD14/MYD14 second-level standard data product of Henan Province from 2008 to 2019, which is a total of 12 years. Therefore, we discuss the temporal and spatial distribution trend of thermal anomalies in Henan Province.

\subsection{Study area}

As a major agricultural province, Henan Province is located at $31^{\circ} 23^{\prime} \sim 36^{\circ} 22^{\prime}$ north latitude and $110^{\circ} 21^{\prime} \sim 116^{\circ} 39^{\prime}$ east longitude. It is located in the Huanghuaihai Plain and is a typical crop planting area

\footnotetext{
"Wenju Shen: gq@henu.edu.cn
} 
with two crops a year. The total area of the province is $167,000 \mathrm{~km}^{2}$, accounting for $1.7 \%$ of the total area of the country. However, the population density is high, with a permanent population of 94.8 million, accounting for $7 \%$ of the total permanent population of the country 7 .

\subsection{Data sources}

This study chose to download the standard product MOD14/MYD14 of MODIS from 2008 to 2019 in Henan Province. This product is mainly for monitoring thermal anomalies-fires on a global scale. It's spatial resolution is $1 \mathrm{~km}$, and it can achieve a monitoring frequency of twice a day under the condition of double-star coordination.

\subsection{Data processing}

\subsubsection{Extraction principle of thermal abnormalities} According to Planck's law, any object whose absolute temperature exceeds $0 \mathrm{~K}$ will radiate energy in the form of electromagnetic waves. When the temperature is constant, the black body emits and absorbs the same electromagnetic wave energy. This law can be expressed by the Planck equation8:

$$
B_{\lambda}=\frac{C_{1}}{\lambda^{5}\left(\exp \left(\frac{C_{2}}{\lambda T}\right)-1\right)}
$$

In the equation, $C_{1}$ and $C_{2}$ are physical constants,
$T$ is the Kelvin temperature, and $\lambda$ is the wavelength. Equation (1) is the physical basis for inversion to obtain the surface temperature, and the Wien displacement law derived from equation (1), equation (2) is the theoretical basis for selecting thermal anomaly remote sensing spectral bands9. The thermal anomaly point information of satellite remote sensing such as MODIS is mainly extracted and calculated based on the Wien displacement law:

$$
\lambda_{m} \times T=2897.9(\mu m \times k)
$$

Where $\lambda_{m}$ is the center wavelength corresponding to the maximum blackbody radiation power at the temperature $T$, and $k$ is the temperature unit of Kelvin. It can be obtained from the equation (2) that the peak value of the energy flux density of the blackbody electromagnetic radiation is inversely proportional to the temperature of the blackbody 10 . Therefore, the threshold can be judged based on the difference between the fire pixel and the background pixel, so as to reach the number of thermal anomaly points.

1.3.2 Thermal abnormality statistics MODIS standard products provide parameters such as fire point reliability, day/night identification code, and surface classification, so that it is convenient for relevant researchers to screen the fire point data under different conditions. At present, this study does not set relevant screening conditions, and aims to analyze the overall situation of thermal anomalies in Henan province from 2008 to 2019.

\section{Analysis}

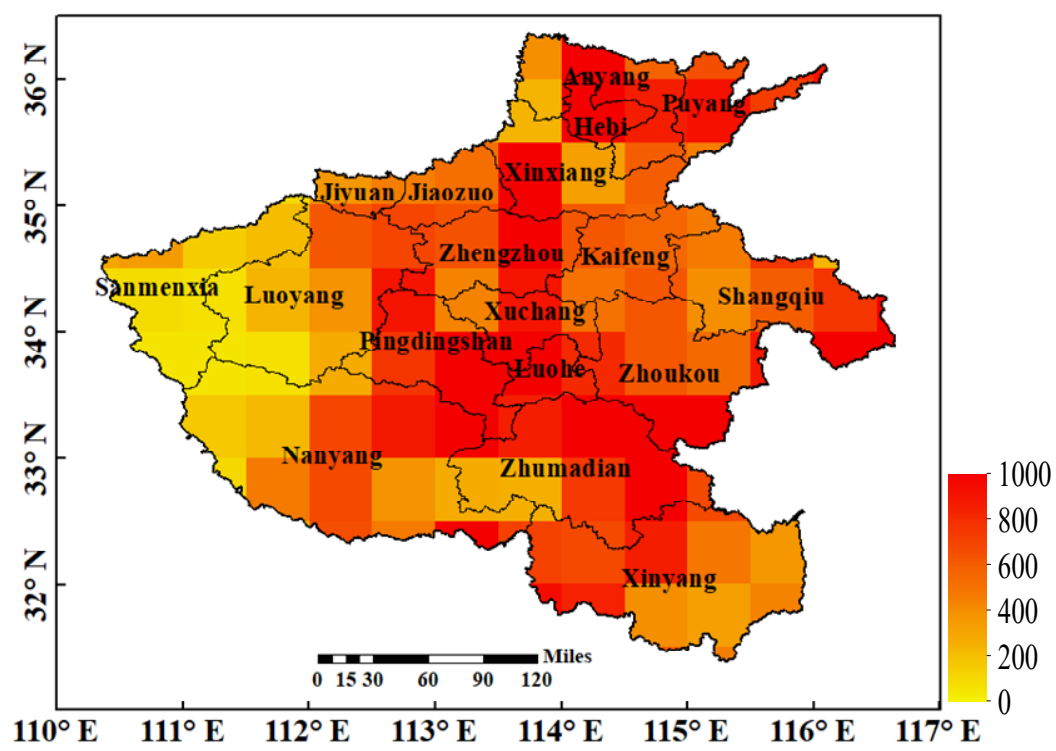

Figure 1. Spatial distribution of thermal anomalies in Henan Province from 2008 to 2019. 


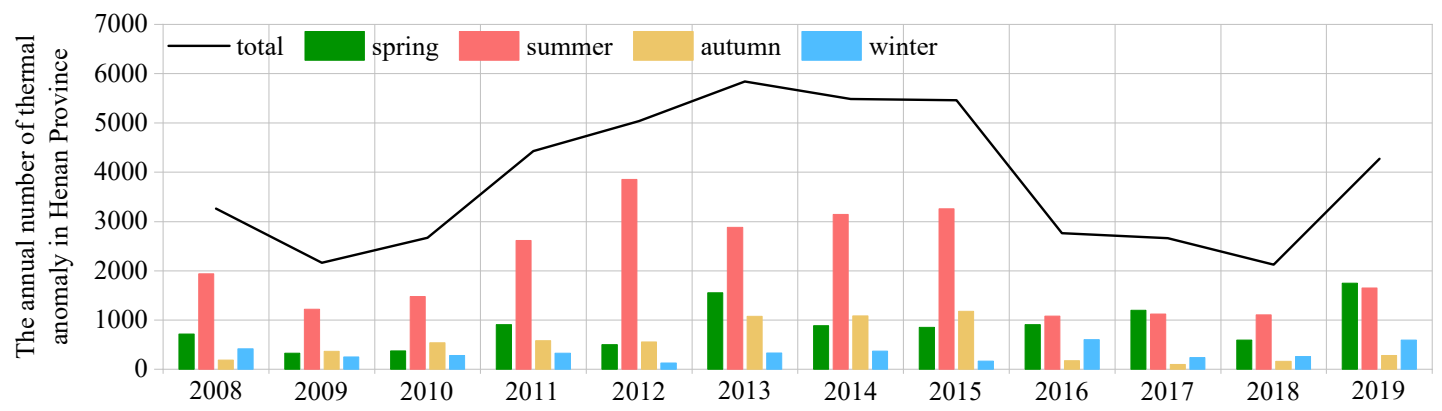

Figure 2. Seasonal and inter-annual changes in the number of thermal anomalies in Henan Province from 2008 to 2019.

Using the MODIS thermal anomaly product data from 2008 to 2019 , a total of 46,176 thermal anomaly points were monitored in Henan Province. Figure 1 shows the spatial distribution of thermal anomalies in Henan Province from 2008 to 2019, and Figure 2 shows the trend of the inter-annual number of thermal anomalies in Henan Province from 2008 to 2019. From Figure 1 and Figure 2 comprehensive comparison found that the overall thermal anomaly points are concentrated in Luohe, Zhumadian, Pingdingshan, Puyang, and Shangqiu. In addition, Anyang, Hebi, Nanyang and Xinyang city also have a relatively concentrated number of thermal abnormalities.

We can clearly see the seasonal and inter-annual changes of the thermal anomaly from Figure 2. Compared with other seasons, the number of thermal anomalies in
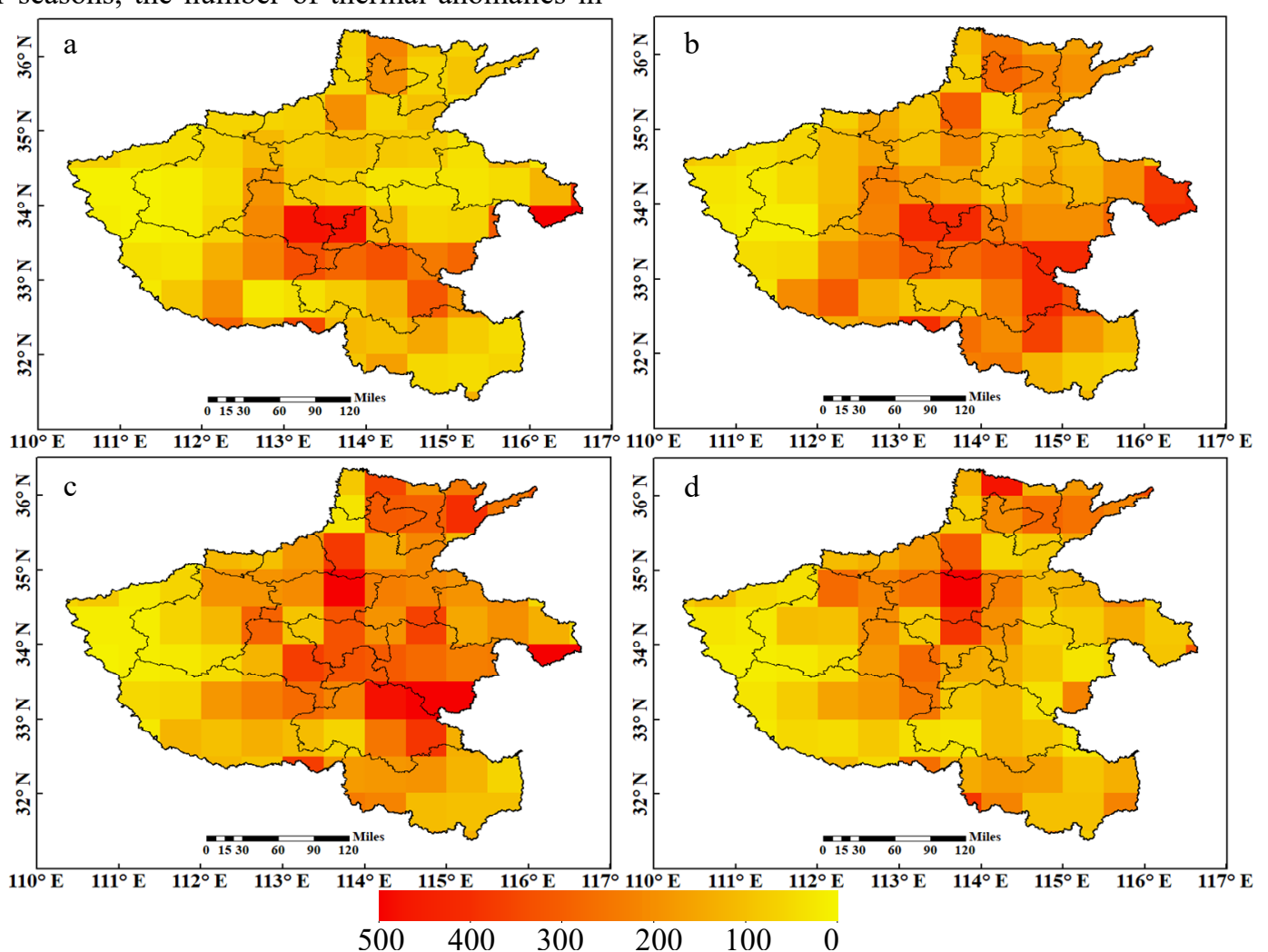

summer is the largest, and the distribution of thermal anomalies is the most obvious; The number of thermal anomalies in winter is the least, and it is the same all year round. In terms of inter-annual changes, the number of thermal anomalies decreased from 2008 to 2009 , and the number of thermal anomalies continued to increase from 2009 to 2013, with an average annual growth rate of $28.3 \%$, and the number of annual thermal anomalies peaked at 5,843 in 2013. From 2013 to 2018, it continued to decline, with an average annual decline rate of $18.4 \%$, and then the number showed an upward trend from 2018 to 2019. These are closely related to the implementation of the burning ban policy and regional characteristics 11 .

Figure 3. Spatial distribution of annual mean values of thermal anomalies in Henan Province from 2008 to 2019(a: 2008 2010; b: 2011 2013; c: 2014 2016; d: 2017 2019).

From the spatial distribution of the annual mean value of thermal anomalies in Henan Province in Figure 3, it can be seen that the number of thermal anomalies in Henan Province from 2008 to 2010 was relatively small, mostly 
and Zhoukou city also had a large number of thermal anomalies. From 2014 to 2016, there has been a continuous increase in the number of thermal anomalies compared to the previous period. Except for Sanmenxia, Luoyang, Nanyang, and Xinyang City, which have fewer thermal anomalies, other regions have a large number of thermal anomalies. From 2017 to 2019, the number of thermal anomalies in Henan Province decreased significantly, and only in some areas of Anyang, Xinxiang,
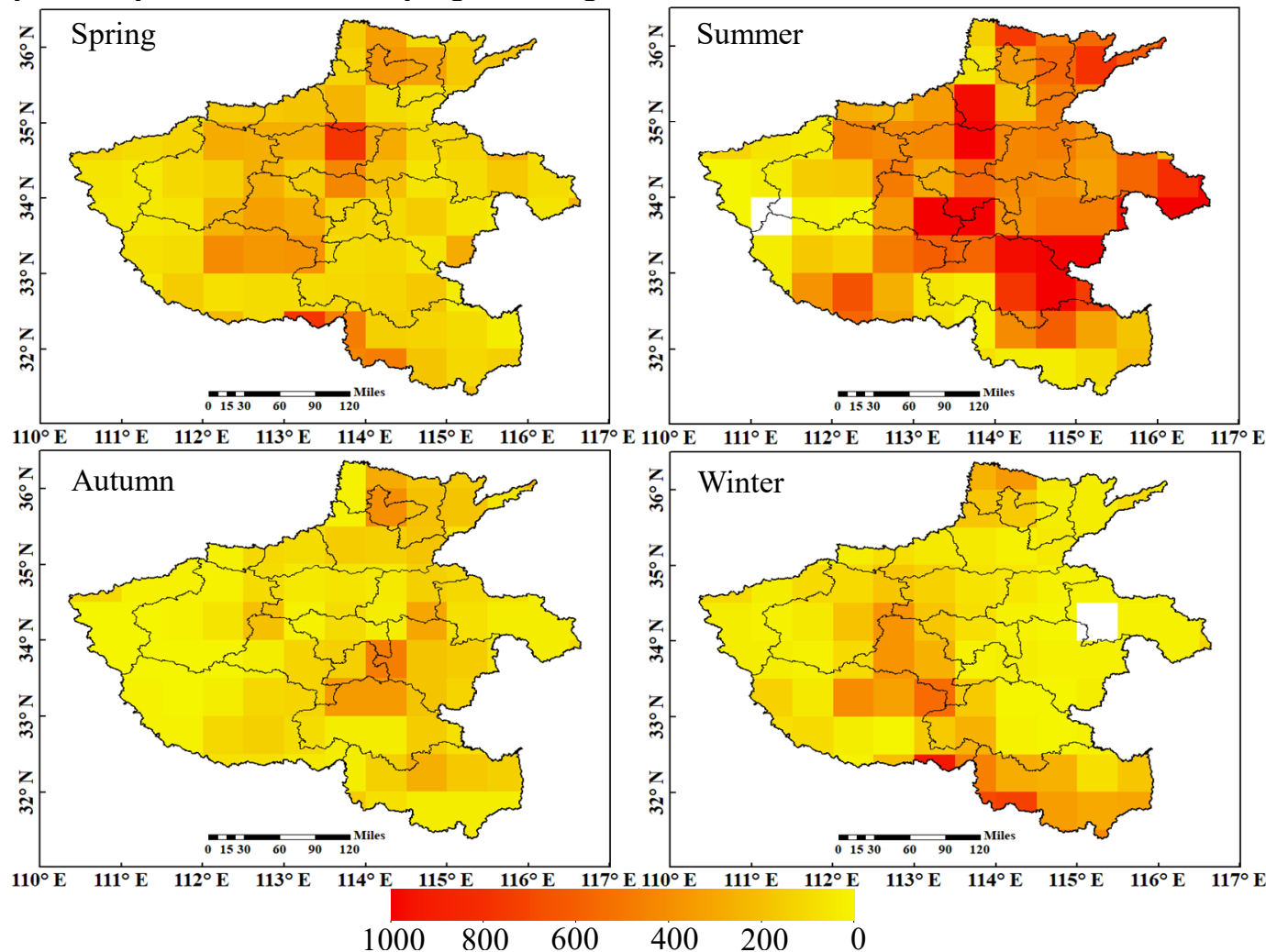

Figure 4. Distribution of the seasonal mean value of the number of thermal anomalies in Henan Province from 2008 to 2019 (Spring (3 to 5), Summer (6 to 8 ), Autumn (9 to 11 ), Winter (12 to 2$)$ ).

Figure 4 shows the seasonal average distribution of heat anomalies in Henan Province from 2008 to 2019. In general, there are relatively more heat anomalies in summer, 25361. The distribution is less in spring, autumn and winter, with 10565, 6276 and 3974 respectively. Summer thermal anomalies are mainly distributed in Zhumadian, Pingdingshan, Puyang, Shangqiu city. The number of thermal anomalies in autumn and winter is small and scattered, and the number of thermal anomalies in winter is relatively more distributed in the mountainous areas of Nanyang and Xinyang city.

\section{Conclusion}

After extracting thermal anomalies from the MOD14/MYD14 secondary standard data products received on the ground, we discusses the temporal and spatial distribution of thermal anomalies in Henan Province from 2001 to 2018 . The results show that:

From 2008 to 2019, the thermal anomaly spots in Henan Province were concentrated in Luohe, Zhumadian, Pingdingshan, Puyang, and Shangqiu city; during the inter-annual changes, the number of thermal anomalies
Zhengzhou and Xuchang city have thermal anomalies. In order to curb the phenomenon of straw burning, the Henan Provincial Government held a video and telephone conference in Zhengzhou on September 22, 2015. The content of this meeting is an important factor leading to the inter-annual change trend of the above-mentioned thermal anomaly. 


\section{References}

1. Sun S, Li L J, Zhao W J, Wang L L, Qiu J, Jiang L, Zhang L K. (2019) Industrial Pollution Emissions Based on Thermal Anomaly Remote Sensing Monitoring: A Case Study of Southern Hebei Urban Agglomerations, China. China Environmental Science, 39(07): 3120-3129.

2. Jin Q F, Wang W H, Ma X Q, Yang S Y, Guo F T. (2017) Temporal and Spatial Dynamics of Pollutants Emission from Forest Fires in Fujian during 20002010. China Environmental Science, 37(02): 476-485.

3. Yang X J, Ma Y F, Peng X J, Guo L F, Cai Q J, Guo F T. (2018) Dynamic changes of pollutants released from forest fire in Southern forested region during 2000-2016. China Environmental Science, 38(12): 4687-4696.

4. Verma S, Dar J A, Malasiya D, Kumar Khare P, Dayanandan S, Latif Khan M. (2019) A MODISBased Spatiotemporal Assessment of Agricultural Residue Burning in Madhya Pradesh, India. Ecological Indicators, 105.

5. He L M, Wang W J, Wang Q, Wei B, Li Q, Wang C Z, Liu X M. (2007) Evaluation of the Agricultural Residues Burning Reduction in China Using MODIS Fire Product. Environmental Monitoring in China, (01): 42-50.

6. Yu K, Qiu L, Wang J J, Sun L, Wang Z M. (2017) Winter Wheat Straw Return Monitoring by UAVs Observations at Different Resolutions. International Journal of Remote Sensing, 38: (8-10).

7. National Bureau of Statistics of the People's Republic of China, (2010). Communiqué on Major Data of the Sixth National Census in 2010 (No.2) [EB/OL]. http://www.stats.gov.cn/tjsj/tjgb/rkpcgb/qgrkpcgb/20 1104/t20110429_30328.html.[2014-10-28].

8. Li Z L, Duan S B, Tang B H, Wu H, Ren H Z, Yan G J, Tang R L, Leng P. (2016) Review of Methods for Land Surface Temperature Derived from Thermal Infrared Remotely Sensed Data. Journal of Remote Sensing, 20(05): 899-920.

9. Peterson D, Wang J, Ichoku C, Hyer E, Ambrosia V. (2013) A Sub-Pixel-Based Calculation of Fire Radiative Power from MODIS Observations: 1: Algorithm Development and Initial Assessment. Remote Sensing of Environment, 129: 262-279.

10. Xu Q Y, Gu W W, Xie T, Liu R. (2017) CropStraw Fire Remote Sensing Monitoring and Its Algorithm Implementation. Remote Sensing Technology and Application, 32(04): 728-733.

11. Sun Y Q. (2018) Analysis of farmer's straw utilization behavior and the burning ban policy's effect - Based on the investigation of 2266 farmers in Henan. Zhengzhou: Henan Agricultural University. 